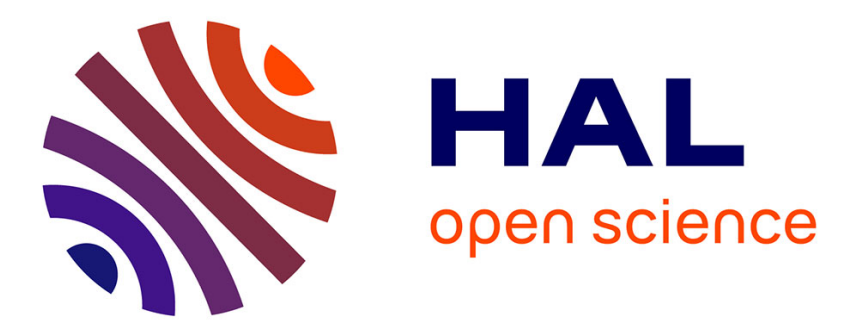

\title{
Les pratiques de partage des connaissances d'une unité de recherche pluridisciplinaire en interne et externe
}

Marie-Claude Bouhedi

\section{To cite this version:}

Marie-Claude Bouhedi. Les pratiques de partage des connaissances d'une unité de recherche pluridisciplinaire en interne et externe. Communication et Management, 2017, 1, pp.69-86. hal-01710354

\section{HAL Id: hal-01710354 \\ https://hal.science/hal-01710354}

Submitted on 26 May 2020

HAL is a multi-disciplinary open access archive for the deposit and dissemination of scientific research documents, whether they are published or not. The documents may come from teaching and research institutions in France or abroad, or from public or private research centers.
L'archive ouverte pluridisciplinaire HAL, est destinée au dépôt et à la diffusion de documents scientifiques de niveau recherche, publiés ou non, émanant des établissements d'enseignement et de recherche français ou étrangers, des laboratoires publics ou privés. 


\title{
LES PRATIQUES DE PARTAGE DES CONNAISSANCES D'UNE UNITÉ DE RECHERCHE PLURIDISCIPLINAIRE EN INTERNE ET EXTERNE
}

\author{
Marie-Claude BOUHEDI"
}

\begin{abstract}
Résumé: Nous proposons dans cet article, de partir d'une étude sur l'usage et la pratique des outils des Technologies d'Information et de Communication pour aborder le partage des connaissances. Nous verrons que le partage est plus facile pour les connaissances explicites mais reste difficile pour une partie des connaissances tacites, certaines connaissances scientifiques et celles dites artisanales, transmissibles seulement par voie orale, observation ou apprentissage. La démarche qualité pourrait contribuer à capitaliser les connaissances. Le déploiement des outils dans le travail collaboratif ne suffit pas en soi : la communication reste essentielle, elle permet d'établir la confiance, qui favorise la coopération.
\end{abstract}

Mots-clés : Information, travail collaboratif, connaissances, communication, savoirs, pluridisciplinaire, TIC, laboratoire, travail scientifique.

\author{
Kwnoledge sharing practices \\ of a multidisciplinary research unit \\ internally an externally
}

Abstract: We propose in this paper, from a study on the use and practice of information technology and communication tools, to address issues of knowledge sharing. We will see that sharing is easier for explicit knowledge but it is difficult for some of the tacit knowledges, some scientific knowledges and the so-called crafts, transmitted only orally, by observation or learning. Quality approach could help to capitalize knowledge. The deployment tools in collaborative work is not enough in itself : communication remains essential, it helps to build trust, which promotes cooperation.

Keywords: Information, collaborative working, communication, knowledge, multidisciplinary.

* Institut National de Recherche Agronomique Centre de Provence Alpes-Cotes d'Azur-Unité de Recherche Ecologie des Forêts Méditerranéennes.s 


\section{INTRODUCTION}

La science est une activité axée sur la production de connaissances, son rôle est de comprendre et d'expliquer les phénomènes. Le partage des connaissances est donc une nécessité et se trouve même ancré dans la tradition du travail scientifique puisque la recherche a pour but de comprendre et découvrir davantage dans son domaine disciplinaire, sans autre objectif que celui de comprendre afin, sous certaines conditions, de pouvoir prédire. Le partage des connaissances et la capitalisation sont des éléments centraux de l'élaboration scientifique, et de la co-construction des connaissances et savoirs. De nos jours, l'introduction des technologies de l'information et de la communication dans les activités au sein des organisations a transformé les modes de travail en travail virtuel ce qui conduit à envisager une équipe classique en équipe virtuelle à degré variable (Griffith et al., 2003; Martin et al., 2004). M. Karoui et A. Dudezert (2010) affirment que "comprendre le contexte de collaboration, c'est aussi approfondir la comprébension du contexte de partage des connaissances et de l'information au sein d'équipes virtuelles» et proposent d'analyser la collaboration selon les points de vue technologique et managérial. Selon les auteurs, les pratiques de travail collaboratif, au sein des organisations, se trouvent de plus en plus orientées vers le partage des connaissances et de l'information. Pour être efficace, la collaboration doit s'appuyer sur une adaptation des outils utilisés pour chacune des tâches à accomplir en fonction du type d'information véhiculée (écrite, verbale ou corporelle) et des modes d'échange en fonction du temps (outils synchrones, asynchrones, application collaborative, GED, etc.) et du mode de structuration de l'information. Ces outils doivent se conformer aux dispositifs managériaux qui offrent un cadre et un guide d'utilisation des outils mais permettent aussi d'établir «un contexte partagé (climat de confiance et de cohésion du groupe). » La collaboration doit également prendre en considération le contexte spécifique à chaque équipe "degré de virtualité, complexité des tâches à effectuer, taille de l'équipe, cycle de vie de l'équipe» et le contexte de partage des connaissances spécifiques à l'équipe (culture de partage des connaissances, familiarité des acteurs avec les technologies de partage des connaissances et de l'information ou les stratégies de partage des connaissances, ou encore des modèles mentaux partagés).

Nous proposons ici d'étudier le partage et la capitalisation des connaissances au sein de l'Unité de Recherche Écologie des Forêts Méditerranéennes (URFM) de l'Institut National de Recherche Agronomique du centre Provence Alpes Côte-d'Azur (INRA PACA), Unité pluridisciplinaire. Bien que ses activités soient pluridisciplinaires, nous avons pu constater, à travers une série d'entretiens que nous avons effectuée auprès des acteurs au sein de l'Unité même, que les connaissances se partagent. Une analyse, plus approfondie, a révélé que l'apport des technologies de l'information et de la communication y contribue favorablement. En outre, la communauté scientifique n'a pas encore totalement adopté les nouvelles formes de communication que ces outils lui offrent.

Dans la littérature, la peur des outils est souvent évoquée lors de la mise en place d'un nouveau système informatique (Guichard, 2002).

Concernant le partage, I. Fabre et C. Gardiès (2008) ont réalisé une étude sur les pratiques informationnelles dans une communauté scientifique et ont mis en évidence des pratiques individuelles et un partage de l'information essentiellement dans la «sphère relationnelle» du chercheur avec des stratégies induisant des rapports de force entre "pairs-concurrents". Selon O. Belin, dont les travaux sont axés sur les problématiques d'introduction des technologies de partage des connaissances dans les organisations, l'organisation peut être vue «comme un lieu communicationnel par excellence» où «les 
enjeux, les jeux de pouvoirs, les cultures, les contextes y sont exacerbés»; mobiliser, de façon complémentaire, des concepts de l'analyse stratégique et de l'analyse contextuelle comme celui du pouvoir ou de l'affordance (des significations), permet de rendre compte des phénomènes organisationnels (Belin, 2007).

On peut interroger alors, pour notre cas, les stratégies mises en œuvre au sein de l'Unité, pour partager les connaissances essentielles à la co-construction des connaissances et savoirs. Comment le partage s'opère-t-il ? Comment partage-t-on et qu'est-ce que l'on partage? Quels sont les outils utilisés? On veut bien partager mais dans quelle mesure et avec qui? Quels sont les enjeux? Comment fait-on avec les documents en interne et les outils existants?

Parmi les hypothèses formulées, nous avons évoqué la réticence à utiliser certains outils au profit de certains moins efficaces en termes de partage, l'embarras du choix face à la multitude d'outils proposés et des habitudes propres à chacun.

Notre objectif était de dégager quelques pistes à explorer et de présenter des recommandations et propositions de stratégies d'information et de communication qui pourraient amener à faciliter la capitalisation des connaissances et à favoriser le processus de partage au sein de l'Unité. Nous aborderons dans un premier temps les approches théoriques afin d'expliquer l'ensemble des phénomènes, ensuite, après une présentation de la méthodologie de recherche, nous présenterons les résultats et discussions qui en découlent.

\section{LES CONNAISSANCES THÉORIES ET CONCEPTS}

\subsection{Savoir et connaissance}

La connaissance a fait l'objet de plusieurs définitions d'auteurs de différentes disciplines. Différente de la donnée, et, plus pérenne que l'information qui est "une donnée ou un ensemble de données articulées de façon à construire un message qui fasse sens. " (Ferrary, Pesqueux, 2006). La connaissance, en science de l'information et communication, est une activité intellectuelle, une construction de l'esprit, qui permet d'appréhender, d'analyser des faits ou des phénomènes afin de les comprendre, de les prévoir, de les capitaliser ou de les reproduire (Meyriat, 1985). Elle est considérée et présentée comme une prise de recul personnelle ou collective par rapport à l'information. La connaissance n'est pas figée, elle se construit en permanence, elle est à la fois durable et diffuse mais le support n'est pas identifiable.

La connaissance et le savoir sont des termes très proches dont la distinction n'est pas facile à faire. J.Y. Prax (2000) définit le savoir «comme un su, quelque chose d'acquis» qui indique «une certaine pérennité, une appropriation qui n'est pas sensée s'oublier ou se perdre, il y a dans le savoir de la certitude et du définitif.» Le savoirfaire et savoir-être sont des dérivés du savoir. L'un lié à la notion de compétence, de l'expérience, des connaissances pratiques mobilisées pour réaliser une activité. L'autre fait référence à la notion de comportement, de conduite ou d'habileté appropriée à l'emploi tel que l'initiative, l'esprit critique, l'autonomie, etc.

La connaissance se définit, ici, comme la capacité d'un individu à analyser et comprendre une information, selon ses aptitudes à apprendre et à mémoriser, pour l'assimiler et générer une représentation personnelle tacite ou explicite avec l'intention d'agir dans un contexte défini.

La connaissance organisationnelle est la faculté à donner aux informations reliées, un sens au sein de l'organisation. Les connaissances organisationnelles sont «un ensemble de connaissances individuelles, spécifiques ou partagées " (Reix, 1995). R. Reix considère que les connaissances sont propres à l'individu et utilise l'expression «les connaissances dans l'organisation». 
En sciences de gestion, de nombreux travaux ont également été menés sur la connaissance au sein des organisations. Selon la théorie de la création de connaissance, (Nonaka et al., 1995) les connaissances surgissent d'une interaction entre deux types de connaissances: les connaissances explicites, bien articulées, stockées sur des supports écrits, et les connaissances tacites, non codifiés, voire non codifiables.

Le processus de création s'effectue au travers d'un cycle de conversions successives des connaissances tacites et explicites, nommé «la spirale de création des connaissances " où les connaissances tacites créées et mobilisées sur le plan individuel sont amplifiées par le biais de quatre modes de conversion des connaissances. - la socialisation (tacite vers tacite) correspond au partage des savoirs faire à travers des échanges collectifs et des expériences partagées au sein d'un groupe, par l'apprentissage par contact direct avec l'expert ou par observation;

- l'externalisation (tacite vers explicite) est la phase d'extériorisation, elle consiste à convertir les connaissances tacites (collective ou individuelle) en connaissances explicites, partagées par orale ou par écrit, compréhensibles par les autres (concepts, modèles, etc.);

- la combinaison (explicite vers explicite) consiste à articuler les connaissances explicites par différents moyens comme les réunions, les documents, les réseaux. C'est la diffusion des connaissances explicites déjà existantes (Alavi et Leidner, 2001), la mise en partage pour recombiner l'information comme les travaux de base de données, statistiques utiles à la décision;

- l'intériorisation (explicite vers tacite) correspond à l'apprentissage, à la transformation du savoir vers le savoir-faire, où les connaissances explicites transmises (formalisées sur supports ou verbalisées) sont assimilées par les individus qui acquièrent de nouvelles connaissances. C'est l'expérimentation, la formation où les connaissances nouvellement créées, modélisées rentrent dans un nouveau cycle de création de connaissances.

\subsection{La production des connaissances scientifiques et I'innovation}

Le partage des connaissances et la capitalisation sont des éléments centraux de l'élaboration scientifique, et de la co-construction des connaissances et production de savoirs. Cependant, la sociologie des sciences, distingue deux modèles relatifs au rapport entre les activités de production de connaissances scientifiques et leur contexte social; le modèle internaliste, basé sur l'importance du travail entre les chercheurs (où la science est autonome et la production de connaissances scientifiques se fait dans le cadre précis des communautés scientifiques), et, le modèle externaliste, basé sur l'importance du rôle principal du contexte social, culturel et historique, sur l'organisation, le mode de fonctionnement mais aussi sur la production des connaissances scientifiques.

Or, comme le souligne P. Bourdieu, les champs scientifiques sont le lieu d'affrontement et de lutte de concurrence, pour l'accumulation de crédit scientifique et pour le contrôle de la science (Bourdieu, 1976). Les connaissances sont des ressources que le scientifique échange sur une sorte de marché contre du crédit scientifique qu'il peut ensuite réinvestir pour produire de nouvelles connaissances et gagner encore plus de crédit mais celles-ci n'ont pas de valeur en elles même; leur valeur tient au fait de pouvoir être échangées contre d'autres biens et dépend de l'importance que les autres accordent à l'objet échangé.

De nos jours, le mode de fonctionnement de l'activité de recherche se déroule telle que le décrit B. Latour (1995) dans la théorie du cycle de construction de la crédibilité, avec la compétition pour le financement et la reconnaissance. Ce qui constitue un paradoxe, puisqu'en contexte 
de compétition il n'y a pas de place pour la transparence qui est une des conditions essentielle à favoriser l'apprentissage, la coopération et le partage des connaissances.

\section{LE PARTAGE DES CONNAISSANCES}

\subsection{Le concept du partage des connaissances}

Nous ne trouvons pas de définitions précises qui fassent l'unanimité. M. Grundstein et C. Resenthal-Sabroux (2001) définissent le partage des connaissances comme le processus de «faire évoluer des connaissances tacites de chacun et construire une connaissance explicite commune au cours d'une interaction entre deux ou plusieurs personnes."

Pour notre étude, le concept de partage des connaissances est "un processus réciproque d'échange des connaissances" qui comprend le partage des deux types de connaissances: les connaissances explicites et les connaissances tacites converties en explicites (Renzl, 2008).

\subsection{La gestion des connaissances}

En s'appuyant sur des travaux sur les connaissances organisationnelles, M. Alavi et D. Leidner (2001) proposent d'introduire des instruments intégrant les différents aspects de la connaissance organisationnelle pour une gestion efficiente de celle-ci : un système de gestion des connaissances. Pour ces auteurs la connaissance organisationnelle peut-être capturée et gérée comme un bien, «un objet » au moyen de dispositifs de gestion adaptés. L'enjeu de la gestion de la connaissance se trouve dans la jonction entre l'individu, les connaissances, et les technologies de l'information : dans la circulation de l'information, la mise à disposition des connaissances, le partage. La connaissance est indissociable de la mise en œuvre, elle est liée à la pratique d'où les théories des communautés de pratiques (Wenger, 1998). La gestion des connaissances favorise le travail collaboratif entre les acteurs au sein d'une organisation et de groupes structurés, la production de nouvelles connaissances et, par là même, le développement de nouvelles compétences au niveau individuel et collectif (Genelot et Lefevre, 2000).

\subsection{Le rôle de la publication au sein de la communauté scientifique}

Les activités de publication, de discussion, de documentation et d'édition sur le web ont favorisé d'autres formes de communication comme l'auto-publication, la participation, la création de réservoirs d'informations et d'échanges chez les internautes. Cependant, selon les premières observations constatées, la communication scientifique et ses processus d'évaluation évoluent lentement et montrent un certain conservatisme (Chartron, 1997). Depuis, d'autres d'études réalisées, entre 1997 et 2004, montrent que de nouvelles pratiques informationnelles et de diffusion de résultats ont été adoptées mais varient selon la discipline et le profil de l'individu (Bégault, 2007).

Parallèlement à ce mode de publication classique, le libre accès se présente alors comme une alternative, avec la mise en place d'archives ouvertes sur Internet et la création de revues gratuites en ligne. Ces nouvelles formes de publication sont en quelque sorte une réponse en réaction aux contraintes que représente le processus de publication traditionnelle, les contraintes éditoriales, le coût d'accès aux revues scientifiques, et l'avènement d'internet; elles représentent un nouveau mode de diffusion des travaux scientifiques et d'échanges, plus proche des besoins du scientifique comme le modèle de l'open-access (Pignard Cheyle, 2005). 


\section{3. ÉTUDE DE CAS: L'URFM}

\subsection{L'URFM du centre de recherche INRA PACA}

L'URFM, terrain de cette étude, mène des recherches pluridisciplinaires en écologie pour une gestion durable des écosystèmes forestiers méditerranéens. L'Unité mène ses travaux autour de trois axes de recherches : biologie des populations et évolution (arbres et insectes), écologie fonctionnelle et dynamique des communautés et physique et écologie du feu. L'URFM applique ses recherches dans les domaines de la gestion forestière, de la conservation des ressources génétiques, de la prévention des incendies et de la protection contre les ravageurs.

\subsection{Méthodologie}

L'étude sur les pratiques de partage des connaissances, réalisée au sein de l'URFM, repose sur une méthode quali-quantitative utilisant de manière bien distincte et complémentaire un questionnaire quantitatif couplé à une enquête qualitative basée sur des entretiens semi-directifs de 30 à 45 minutes, et, compte-tenu de notre statut de permanent dans l'URFM, en adoptant une attitude d'observation participante complète au cours des réunions. Comme le préconise A. Blanchet et al. (2010) l'enquête par entretien est conçue pour apporter une information biographique et se caractérise, contrairement au questionnaire, par une forme moins contraignante : elle est exploratoire. "L'entretien est l'instrument privilégié de l'exploration des faits dont la parole est le vecteur principal.» Ces faits concernent les systèmes de représentations et les pratiques sociales.

Cette enquête s'est déroulée sur deux années. Les entretiens réalisés en 2012 au sein de l'URFM ont été, enregistrés et retranscrits, puis, repris l'année suivante pour une étude plus approfondie et enrichie. Cette approche paraissait intéressante pour identifier les pratiques usuelles de chaque individu, concernant le partage de connaissance, connaître son vécu et son point de vue sur le partage dans l'organisation mais il était aussi nécessaire pour construire le questionnaire. En effet, ce dernier permet de distinguer les catégories d'individus selon leur statut socioprofessionnel et leur tranche d'âge, de dessiner un panorama, et d'apporter des éléments de réponses.

Ces méthodes d'enquête se complètent, l'analyse quantitative permet de mesurer les faits. L'analyse qualitative peut révéler des stratégies individuelles, aussi complexes qu'elles soient, mais nécessite un recours aux données quantitatives pour préciser s'il s'agit d'un comportement marginal ou général. Cependant, le recoupement avec des informations et observations complémentaires est nécessaire pour valider les données et résultats obtenues.

L'analyse de contenu des récits, au moyen d'une grille, nous a permis de relever des redondances et des séquences révélant des représentations et d'identifier ainsi des thèmes comme la nature des connaissances partagées, la classification des outils utilisés, la culture de partage. Elle a aussi permis d'émettre des hypothèses et d'élaborer les questions plus ciblées du questionnaire pour nous renseigner sur les types de connaissances partagés, la pratique et l'utilisation des outils, les freins au partage, l'intérêt de partager.

L'enquête en ligne a permis de confirmer les résultats obtenus lors des entretiens et de fournir une meilleure compréhension des pratiques du partage et des usages des outils au sein de l'Unité.

Cependant, en raison des nombreux déplacements des agents, vingt et une interviews ont été réalisées sur trente-six agents ciblés : cinq chercheurs sur onze, huit doctorants sur huit, quatre ingénieurs/assistants sur six et quatre techniciens sur onze.

Le questionnaire, élaboré à partir des résultats et analyses des interviews puis testé et corrigé, a été mis en ligne active en juillet 2013, durant 24 jours et diffusé aux chercheurs, ingénieurs et 
techniciens de l'unité. Il a permis de recueillir, après 4 relances, les réponses complètes de 27 agents sur 43 , soit $62,79 \%$ de l'effectif global. Ce pourcentage est significatif, il correspond au taux de retour et à la représentativité de l'échantillon attendu lors d'une enquête auto-administrée.

Les entretiens semi directifs ont amené les interviewés à révéler les pratiques et les moyens utilisés pour l'acquisition des connaissances et le transfert de ces connaissances. Ils ont permis d'établir un état des lieux des connaissances partagées, des moyens et activités mis à disposition pour le partage des connaissances, puis d'entrevoir l'état d'esprit des interviewés vis-à-vis de l'organisation sur le partage. Nous ré-exploitons ces données, ici, dans le but de tenter une analyse sur les pratiques de partage de connaissances et les outils utilisés.

En revanche, notre position n'étant pas neutre, on pourrait émettre certaines réserves et considérer qu'elle ait pu influencer les discours : l'autocensure, la prise de parti, la réclamation, etc. En effet, il n'est pas aisé de concilier une méthodologie en immersion dans la vie d'un groupe avec le recul et la mise en perspective nécessaires au rôle de chercheur (Diaz, 2005). Cependant, on peut aussi considérer que notre implication dans l'Unité a pu être un élément favorable pour appréhender la problématique portant sur les usages de ces chercheurs.

\subsection{Résultats et discussions}

L'échantillon de personnes interviewées, même s'il ne comprend pas la totalité des acteurs de cette Unité de recherche, a permis, cependant, de dresser des profils représentatifs quant à la question qui préoccupe le plus, à savoir le partage des connaissances.

\subsection{Classification \\ des connaissances}

La plupart des participants interrogés ont dit partager les connaissances. Sur les 27 questionnaires en ligne traités, 100\% des répondants distinguent cependant plusieurs types de connaissances parmi lesquels certains sont partagés plus fréquemment que d'autres. Ces types sont présentés dans le tableau ci-dessous. Leurs caractéristiques et les observations ont été reprises telles que les ont formulées et exprimées les interrogés lors des entretiens, 15\% ont répondu partager des données.

Cf. Tableau 4, page 76.

Nous retenons dans cette typologie des connaissances qu'il existe des connaissances faciles à partager et d'autres difficiles, voire très difficiles. Le processus de «socialisation», à savoir, l'échange d'expériences, l'observation, l'imitation, la pratique ou encore le langage (réunions, face à face, etc.) permet le transfert des connaissances. Mais le transfert s'opère aussi par le processus de "l'intériorisation", conversion de la connaissance explicite en connaissance tacite; elle correspond à la phase d'apprentissage. Pour ce faire elle nécessite d'être verbalisée ou présentée sous forme de signes dans un document (Nonaka et al., 1997).

Or, $56 \%$ des répondants estiment qu'il n'est pas aisé de décrire ce que l'on sait faire et reconnaissent la nécessité d'une formation ou d'un accompagnement. On peut en déduire que ces agents rencontrent des difficultés lorsqu'ils formalisent des connaissances tacites en connaissances explicites. Les connaissances tacites sont des savoirs mais, bien qu'elles soient en partie codifiables et diffusables, il n'en demeure pas moins qu'une autre partie ne l'est pas (Polanyi, 1967). Partager ce type de connaissance ne peut se faire que par les échanges et la mise en relation entre les individus que rend possible la cartographie des connaissances par une représentation visuelle des savoirs des individus ou groupes d'individus liés à un métier (Sellin, 2011). Mais aussi les annuaires des compétences qui, par la localisation de l'expertise, facilitent la mise en relation et permettent les interactions entre les acteurs et de savoir qui fait quoi, ainsi que les échanges d'expériences entres les agents, les apprentissages mutuels et la formation. 
Tableau 4 : Typologie des connaissances évoquées par les répondants à l'enquête sur le partage des connaissances à l'URFM

\begin{tabular}{|c|c|c|c|}
\hline Types de connaissance & Caractéristiques & Observations & $\begin{array}{c}\text { Réponse } \\
\text { en \% }\end{array}$ \\
\hline $\begin{array}{l}\text { Connaissances fondamentales } \\
\text { (scientifiques) }\end{array}$ & $\begin{array}{l}\text { Formation initiale (disciplines, } \\
\text { expertise). }\end{array}$ & $\begin{array}{l}\text { Difficiles à partager, très } \\
\text { difficiles pour la science du } \\
\text { feu }\end{array}$ & $18 \%$ \\
\hline Connaissances générales & Connaissances diffuses. & Partagées & $18 \%$ \\
\hline $\begin{array}{l}\text { Connaissances du réseau } \\
\text { scientifique }\end{array}$ & $\begin{array}{l}\text { Réseaux d'infrastructures } \\
\text { (ICOS, RTE); } \\
\text { Plateforme de modélisation } \\
\text { (Capsis); } \\
\text { Réseaux thématiques (groupe } \\
\text { des entomologistes). }\end{array}$ & Difficiles à partager & $7 \%$ \\
\hline Connaissances techniques & $\begin{array}{l}\text { Connaissances pratiques } \\
\text { (outils statistiques, } \\
\text { métrologie, dendrométrie). }\end{array}$ & Partagées & $19 \%$ \\
\hline $\begin{array}{l}\text { Connaissances du terrain } \\
\text { «artisanales» }\end{array}$ & $\begin{array}{l}\text { Connaissances fiable } \\
\text { (issues de l'observation du } \\
\text { technicien, des collègues } \\
\text { gestionnaire ou naturalistes, } \\
\text { ou passionnés au chercheur); } \\
\text { Connaissances des paramètres } \\
\text { environnementaux (pour } \\
\text { mettre en œuvre et faire des } \\
\text { expérimentations, données). }\end{array}$ & $\begin{array}{l}\text { Difficiles à partager, à faire } \\
\text { le lien avec la science, en } \\
\text { raison de fortes probabilités } \\
\text { d'erreurs factuelles }\end{array}$ & $9 \%$ \\
\hline $\begin{array}{l}\text { Connaissances } \\
\text { bibliographiques }\end{array}$ & $\begin{array}{l}\text { Connaissances liées aux } \\
\text { publications (plus formatées). }\end{array}$ & Partagées & $12 \%$ \\
\hline Autres & $\begin{array}{l}\text { Connaissances } \\
\text { administratives. }\end{array}$ & Partagées & $2 \%$ \\
\hline
\end{tabular}


Tableau 5 : Les types d'outils, dispositifs et fonctions

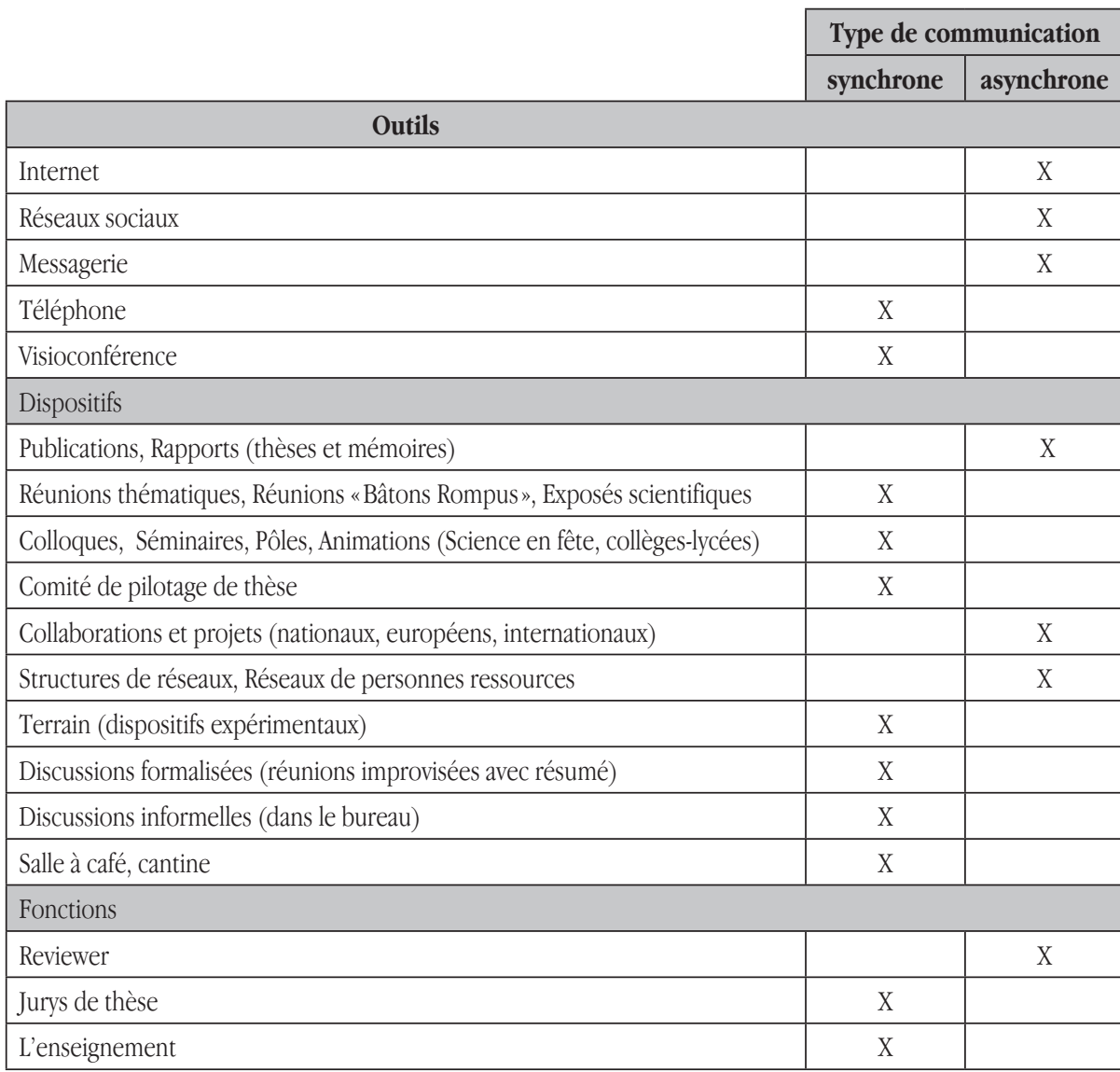

\subsubsection{Les outils et dispositifs} de partage à I'URFM

Au cours de ces entretiens, la majorité des participants interrogés dit partager les mêmes outils. L'analyse de ces interviews nous a permis d'établir un panorama des outils et dispositifs utilisés par l'ensemble des personnes interrogées.

Cf. Tableau 5.

Cependant, ces différents moyens amènent à deux modes de communication qui selon leur modalité d'utilisation peuvent avoir une incidence sur l'échange: le mode de communication synchrone où deux à plusieurs individus communiquent en temps réel, et, le mode de communication asynchrone où la communication est décalée dans le temps. L'intérêt des outils asynchrones est qu'ils offrent une plus grande souplesse temporelle et géographique. Mais ce mode communicationnel est moins riche et ne peut restituer certains aspects du face à face comme la présence, le socio affectif, la synchronisation des échanges, le feedback, etc. 
Les réunions formelles et informelles, les séminaires, sont des moments d'échanges qui facilitent aussi la compréhension des connaissances et savoirs. Ces réunions sont nécessaires, et profitables à l'Unité, comme celles des techniciens, elles permettent de créer des interactions entre agents qui n'auraient pas pu se faire dans le cadre de leurs seules missions. Ce qui favorise la créativité car les agents osent plus volontiers proposer leurs idées et prendre des risques.

Les colloques semblent être le moyen de rencontrer des personnes référentes et de créer son propre réseau. Les réseaux scientifiques sont un bon exemple d'une culture organisationnelle basée sur la collaboration et la confiance. Ils favorisent le partage des connaissances notamment dans le cadre des projets de recherche qui permettent d'échanger des données, mais, plus largement, de structurer, d'organiser et normaliser, chacun dans son domaine d'activité, selon des normes internationales partagées, afin de valider les connaissances sur un plan plus large.

Les communautés de pratiques (Wenger 1998, Fox, 2000) sont des réseaux plutôt fermés, où l'on échange des savoir-faire, des problèmes proches et des pratiques techniques issues d'expériences, tout en offrant des méthodes qui ont été validées, qui ont fait leur preuve, et auxquelles ils peuvent accorder leur confiance.

Notre hypothèse de départ, selon laquelle la grande majorité utilise les outils avec lesquels ils ont l'habitude de travailler, se confirme au vu des résultats recueillis pour le partage, comme la messagerie ou le papier, asynchrones. En outre, les répondants ont souligné l'importance et l'efficacité de l'échange en face à face, par voie orale, "en faisant » sur le terrain, en vidéo, en réunion en présentiel ou visioconférence et l'ont confirmé en optant pour les outils synchrones.
Tableau 6 : Outils et dispositifs utilisés pour le partage

\begin{tabular}{|c|c|}
\hline & $\begin{array}{c}\text { Nombre } \\
\text { de réponses }\end{array}$ \\
\hline \multicolumn{2}{|l|}{ Outils } \\
\hline Messagerie & 24 \\
\hline Diffusion papier & 15 \\
\hline Téléphone & 9 \\
\hline GDo & 4 \\
\hline Silverpeas & 2 \\
\hline Forum & 2 \\
\hline $\begin{array}{l}\text { Archives Ouvertes (HaL, } \\
\text { Prodinra, etc.) }\end{array}$ & 2 \\
\hline Xwiki & 1 \\
\hline Facebook & 2 \\
\hline Google & 1 \\
\hline \multicolumn{2}{|l|}{ Dispositifs } \\
\hline Réunion ou visioconférence & 20 \\
\hline Discussion spontanées & 20 \\
\hline Autres & 2 \\
\hline
\end{tabular}

En revanche, les outils offrant la possibilité de collecter et de partager ne sont pas utilisés de façon équivalente et bien souvent, sont sous-exploités :

Tableau 7 : Utilisation des doubles fonctionnalités

\begin{tabular}{|l|c|c|}
\hline \multicolumn{1}{|c|}{ Outils } & $\begin{array}{c}\text { Pour } \\
\text { recueillir }\end{array}$ & $\begin{array}{c}\text { Pour } \\
\text { partager }\end{array}$ \\
\hline Liste de diffusion & 12 & 24 \\
\hline Facebook & 2 & 0 \\
\hline Forum & 4 & 2 \\
\hline $\begin{array}{l}\text { Archives ouvertes (HAL, } \\
\text { Prodinra, etc.) }\end{array}$ & 9 & 2 \\
\hline Blog & 0 & 0 \\
\hline Autres & 7 & 2 \\
\hline
\end{tabular}

Globalement, les agents citent les outils mis à leur disposition mais ne les utilisent que très peu, 
voire pas du tout, souvent par manque d'information sur ces outils.

La masse d'information sur internet, le manque de lisibilité et de fiabilité, la crainte sur les sources et le manque de temps sont les raisons qui ont été les plus évoquées. Cependant, nous relevons, dans ces résultats, le paradoxe suivant : la plupart des interviewés ont conscience que les outils ont amélioré la rapidité et permis un gain de temps dans la recherche d'information et le partage mais ils ne les utilisent pas car les jugeant chronophages et préfèrent les outils qu'ils ont l'habitude d'utiliser. Ce que I. Fabre et C. Gardiès (2008) ont relevé "les chercheurs développent leur propre système d'information mais, par manque de compétences informationnelles et de disponibilité, simplifient leur pratiques.»

D'autre part, la multiplicité des outils collaboratifs existant au sein de l'Unité déroute la plupart des agents pour qui les tâches de bureautique restent une activité mineure par rapport à leur activité principale. De plus, le mode de fonctionnement de la recherche s'effectue le plus souvent en mode projet, en collaboration avec d'autres partenaires extérieurs, géographiquement plus ou moins éloignés, induisant de nombreuses et indispensables interactions. Pour ces agents, s'adapter et se former à différents outils est ressenti comme un défi. "Or, l'usage de la technique suppose que l'on mette au centre l'acteur social,... et que la technique soit pensée à partir de lui et de ses besoins» (Bernoux, 2009). Par conséquent, le choix d'UNE solution adaptée aux besoins et au contexte des utilisateurs, faciliterait et favoriserait des interactions à distance et différées dans le temps.

Le gestionnaire de documents électroniques GDo est très peu utilisé. Ceci confirme ce qu'a révélé les interviews : la démarche qualité n'est pas adoptée par tous car souvent perçue comme contraignante ou comme cet interlocuteur l'indiquait, une véritable menace "Rationaliser $(A Q R)$ les choses je n'y crois pas car il n'y a rien de mieux que le dialogue. L'AQR risque de remplacer nos techniciens...» Nous relevons ici une «résistance» face à la mise en place de la démarche qualité imposée par le management, ce que E. Friedberg décrit comme «...de l'irrationalité bumaine qui résiste à la rationalité et à la rationalisation des activités incarnées par l'organisation..." (Friedberg, 1997). En sociologie, l'organisation est vue comme un lieu de tension et de conflits où les comportements des acteurs sont liés aux buts et motivations de chacun au sein de l'organisation (Crozier, Friedberg, 1977).

Le changement est un phénomène complexe et délicat à mettre en place «Celui-ci doit se raisonner en termes de pouvoir: celui qui a le sentiment de perdre ne peut que refuser le changement ou tenter de le freiner " (Bernoux, 2009). Pour ce qui concerne l'outil imposé dans la pratique de la recherche, O. Belin, pour son cas, nous dit " [L'intranet] pose la question de l'organisation de la recherche débarrassée des effets pervers de la concurrence sur la structure et sur les laboratoires... mais concurrentielle quand même.» L'outil n'est pas uniquement un moyen de pouvoir de l'organisation pour contraindre mais peut être aussi un moyen de remise en question des «modalités scientifiques» (Belin, 2007). L'activité de partage, et par là, la dissémination de données se heurtent aux logiques de confidentialité et de protection des informations considérées comme des «normes partagées par tous les chercheurs.»

Comme le soulignent $\mathrm{H}$. Abba, et S. LeleuMerviel (2010), dans un contexte de changement d'organisation, notamment lorsqu'il s'agit de s'approprier un outil informatique, la communication, la coopération et la mise en confiance des individus contribuent à lever les résistances au changement.

\subsubsection{La capitalisation des connaissances par la démarche qualité}

Pour justifier auprès de ses partenaires et financeurs, la qualité de ses travaux et des résultats mais 
aussi, pour son propre intérêt, afin de perfectionner ses activités de recherche, l'INRA a élaboré, à l'attention de ses Unités de recherche, un référentiel pour la Qualité. Ce guide, comparable aux normes de type ISO 9001, a pour objectif principal de prévenir des dysfonctionnements qui nuiraient à la réalisation d'un produit ou d'un service conformément aux attentes d'un client. Pour l'INRA, cette garantie des pratiques, dont la qualité est conforme aux normes partagées, constitue un élément de langage commun entre elle et ses partenaires et financeurs mais génère des changements dans le travail comme la formalisation écrite, mais aussi dans le comportement, avec la vigilance accrue pour la détection et l'enregistrement des dysfonctionnements ainsi que la gestion des documents générés (Granier et al., 2009).

Pour ce faire, l'URFM utilise, entre autre, le logiciel GDo, mis à la disposition des Unités depuis 2006. L'apport de cet outil en matière de traçabilité est incontestable et indispensable mais il l'est également pour le partage des connaissances dans le travail collaboratif. Il contribue à la capitalisation des connaissances. En effet, il permet de collecter, de codifier, de stocker afin de pérenniser et de mettre à la portée de tous les agents des savoirs et savoirfaire comme des protocoles, check-list, rapports de projets, procédures techniques et administratives, des données provenant d'expériences et d'analyses scientifiques, avec une traçabilité des travaux de recherche et un référencement au cahier laboratoire et archives numériques. Pour certaines connaissances, au-delà de la garantie qualité, cet outil participe à la démarche d'autoformation. Or il semblerait, bien que de nombreux documents y soient répertoriés, que la démarche qualité soit sous-exploitée et insuffisamment partagée par tous.

D'après D. Vinck et al. (2004), ce sont essentiellement les connaissances liées aux activités techniques et administratives qui ont tendance à être routinières; pour celles-ci la démarche qualité permet d'apporter une structuration et une organisation qui favoriseraient un gain de temps et une meilleure production qualitative. Selon les auteurs, les activités de recherche comportent des aspects variés et seule la mise en place d'un dispositif adéquat pourrait permettre de gérer, d'assurer le suivi et de capitaliser l'historique des projets de recherche car au-delà de la publication des résultats, les connaissances scientifiques sont difficiles à capturer. Tout au long de leurs projets de recherche, beaucoup de connaissances, (mémoires de projet, tableau de bord, documentation, etc.) qu'ils nomment "artefacts», sont créées et ont contribué à la construction et à la réalisation des travaux avant d'aboutir à des résultats mais, pour lesquels il n'existe pas d'outil qui permettent de les capitaliser. La démarche qualité pourrait être utilisée comme dispositif pour capitaliser ces «résultats intermédiaires.»

Nous retenons ici que la démarche qualité contribue à favoriser la communication et la confiance et, de ce fait, le partage d'une partie des connaissances, les autres, scientifiques, restent difficiles à partager.

\subsubsection{Culture du partage au sein de l'Unité}

Dans leur ensemble, les interviews révèlent une forte culture de partage; plus d'un tiers partage car ils éprouvent un intérêt à en faire profiter la communauté, et 25\% pour apporter leur contribution au savoir. En développant «une forte culture de partage d'information ", la circulation de l'information est d'autant plus importante que celle-ci n'est plus considérée comme une source de pouvoir (Perrin, 2008).

Ainsi, 12 répondants partagent pour une satisfaction personnelle, 8 autres pour avoir un retour et 4 autres pour une reconnaissance personnelle. Dans la communauté scientifique, la relation qui lie les scientifiques se fonde sur le principe du don contre don : on donne pour avoir en contrepartie un retour (Altier, 2009). 
Nous avons relevé que les échanges se faisaient entre pairs, le plus souvent à l'intérieur de chaque groupe : Doctorants/Doctorants, Techniciens/ Techniciens. Les discussions et le partage de connaissances s'effectuent entre scientifiques, il arrive même parfois que des relations paternalistes s'établissent entre le chercheur et les doctorants encadrés, comme pour ce répondant "La non communication des résultats, par peur de se faire voler les données. Du coup, on est plus prudent pour les thésards - de manière inconsciente. Cependant comme «laproduction de données crédibles... est un moyen d'activer le cycle de crédibilité et de mettre en mouvement le «commerce de la science... " (Latour, 1996). La publication scientifique est l'élément indispensable pour le chercheur dans sa quête de légitimité: un article entraîne la reconnaissance par les pairs, génératrice de collaborations potentielles et de subventions. Aussi, cette prudence vise t'elle sa "crédibilité scientifique."

Les techniciens échangent entre eux lors des déplacements sur le terrain où ils peuvent passer quelque fois plusieurs jours ensemble. Un interviewé, en particulier, exprime son ressenti mais qui n'est pas partagé dans l'Unité « $L a$ dichotomie entre techniciens et chercheurs est très forte à l'INRA, ce qui n'est pas le cas au CNRS où le directeur, le chercheur vont sur le terrain. Néanmoins les chercheurs aimeraient bien s'y rendre mais les techniciens n'ont pas besoin d'eux sur le terrain. " Nous nous interrogeons cependant, si ce phénomène relationnel, lié à l'appartenance au groupe, provient d'une conception donnant lieu à un malentendu ou s'il est latent.

Au sein de l'URFM, il existe bien une culture de partage, les agents n'hésitant pas à solliciter

1. Au cours des évaluations de l'Unité en 2007 et 2010, la commission d'évaluation a rencontré les différents personnels qui ont exprimé une forte motivation et adhésion au projet d'Unité mais cette dichotomie n'a pas été évoquée et n'est pas ressortie dans le rapport de l'AERES. leurs collègues, à s'impliquer dans l'animation de groupes de travail.

En revanche, certains interviewés ont soulevé un aspect de l'activité de recherche desservant la culture de partage des connaissances et de socialisation au sein de l'Unité, rapporté ici « $L a$ compétition qui encourage toujours à faire différemment plutôt que d'utiliser des méthodes qui marchent et prouvées par ailleurs. ", "La non communication de données c'est plus un problème de personnalité que de stricte confidentialité. » Le manque de temps est l'un des principaux freins au partage, 20 personnes l'ont évoqué : il est un problème prégnant dans l'activité qui ne laisse pas de place à la communication sur les projets en dehors des publications. Comme celle-ci le déplore «Un site qui recense tous ce qui est possible de faire sur modèle va être plus lu par des scientifiques et non scientifiques que 25 publications de 30 pages". Les facteurs liés à l'activité de recherche où la production de connaissances et les évaluations font que les comportements des chercheurs, concernant le partage, sont très variables. Comme le souligne Bourdieu, celui qui publie se retrouve donc dans une compétition permanente pour être reconnu scientifiquement et socialement par ses pairs (Bourdieu et al., 1992).

Aussi, le chercheur est perpétuellement tiraillé entre partager ses résultats par la voie formelle afin de préserver la paternité de ses résultats et partager en dehors de cette voie, ce qui lui permet d'échanger et d'acquérir de nouvelles connaissances.

\subsubsection{Le sens commun au sein d'une Unité de recherche interdisciplinaire}

La grande majorité des agents interrogés a conscience de l'utilité des informations qu'elle possède, excepté 2 personnes. Ainsi, 92\% d'entre eux savent qu'ils partagent des informations utiles mais aussi avec qui les partager. Ces résultats nous 
confirment que le contexte pluridisciplinaire n'est en aucun cas un frein au partage des connaissances entre les agents. Bien au contraire, il semblerait que cet environnement tende à favoriser l'émergence de nouvelles connaissances. Cependant, cette question mériterait d'être approfondie (quels sont ces nouvelles connaissances, de quels types, entre qui et qui etc., afin de recueillir plus d'information et d'en tirer une analyse plus fine des résultats obtenus.

En effet, au sein de l'URFM ${ }^{1}$ des généticiens, des écologistes du feu, des éco-physiciens, des physiciens, entomologistes travaillent plus ou moins sur des projets communs. Or, une discipline possède son propre référentiel qu'elle utilise pour étudier un sujet. Elle élabore son propre langage, et utilise ses propres techniques qu'elle a mises au point grâce à des théories qui lui sont également propres (Morin, 1994). Nous en déduisons que, dans le contexte de l'URFM, la représentation des connaissances doit poser un véritable problème de par la diversité des points de vue des chercheurs. Ce qu'exprime ce répondant "Depuis l'arrivée de François on a élargi dans l'équipe notre domaine de recherche, il a fallu acquérir de nouvelles compétences et des connaissances, chercher d'autres revues, des connaissances fondamentales.»

Dans la littérature, des cas d'étude montrent que des solutions d'organisation, de structuration, de codification, la construction d'ontologies, permettent de relever les défis que posent la pluridisciplinarité face à la gestion des connaissances mais aussi au partage de ces dernières. Depuis 2013, l'Unité participe à la mise en place de référentiels sémantiques (thesaurus, ontologies) dans le cadre de l'infrastructure du

1. L'interdisciplinarité est le fondement du projet scientifique de l'URFM. La plupart des chercheurs de l'URFM sont motivés pour la mettre en œuvre car ils ont conscience de la plus-value qu'elle représente pour l'Unité, mais aussi dans l'évaluation portée par le département sur l'Unité. Mais il y a des freins, notamment ceux évoqués par Edgar Morin. D'où la nécessité de plus d'actions d'échange et de collaboration que dans une Unité mono disciplinaire. projet Anaee France dédié à l'étude des écosystèmes continentaux (Pichot et al., 2015). Il s'agit de la création d'un vocabulaire normé afin d'éviter toute ambiguité, en gérant la synonymie ou la polysémie. Anaee est un projet européen d'infrastructure scientifique dont le but est de remédier à l'actuel morcellement de la recherche sur les écosystèmes en Europe en mettant en place un ensemble coordonné de plateformes expérimentales, des plateformes analytiques, et des systèmes d'informations pour la gestion des données et la modélisation (Anaee-France). Ces outils permettront de tester, analyser et modéliser les réactions des écosystèmes aux changements climatiques et d'élaborer des techniques de gestion appropriées.

À l'issue de l'analyse des contenus, les connaissances se sont avérées globalement bien partagées au sein de l'Unité pluridisciplinaire. Cet aspect avait été identifié comme étant un levier permettant de faire émerger de nouvelles connaissances et favorisant l'interaction entre les chercheurs dans la construction de nouveaux projets. Cependant, l'analyse du questionnaire a permis de mettre en évidence que les outils mis à la disposition du personnel étaient, non seulement mal adoptés par la communauté scientifique, mais aussi sous exploités au profit d'autres avec lesquels les agents étaient plus familiarisés. La démarche qualité pourrait contribuer à capitaliser les connaissances mais nécessiterait des changements de comportement et d'organisation qui ne sont pas adoptés par tous.

\subsection{Propositions et recommandations}

Compte tenu des différents points que nous avons relevés et de nos analyses, nous pouvons émettre quelques suggestions qui pourraient permettre d'améliorer le partage des connaissances et la capitalisation au sein de l'URFM, 
et que nous formulons ici sous forme de liste d'actions à mener.

Au niveau des technologies:

- informer sur les outils, leur intérêt et leur finalité et selon définir les types d'information à diffuser. Certains agents sont plus pionniers que d'autres: s'appuyer sur eux pour faire des retours d'expérience;

- former sur les outils : en dehors de la formation permanente, proposer des tutoriels sous différentes formes;

- nommer des agents qui maîtriseraient bien les outils et qui pourraient jouer le rôle de facilitateurs;

- proposer une plateforme collaborative adapté au contexte des utilisateurs, en mode gestion de projet, comme solution à la multiplicité des outils sans pour autant apporter des contraintes supplémentaires aux utilisateurs ;

- assurer une veille car la technologie évolue rapidement dans ce domaine.

Au niveau managérial :

- répertorier les communautés existantes au sein de l'organisme, développer les pages personnelles en ligne afin d'en établir un annuaire ou/ et une cartographie, de savoir qui participe;

- favoriser les rencontres professionnelles et les rencontres conviviales afin de développer et renforcer le lien relationnel entre les agents ;

- valoriser les pratiques collaboratives, favoriser les initiatives, la créativité et les échanges autour stimuleront le partage des savoirs, l'envie de "faire ensemble» et l'émergence de nouvelles connaissances;

- former à la coopération et la collaboration pour développer des compétences individuelles et collectives;

- identifier des animateurs (par projet) pour mobiliser, créer une dynamique, valoriser, canaliser et contrôler de façon à ce que les agents se déplacent vers ces plateformes collaboratives pour partager;
- répertorier les compétences existantes au sein de l'Unité, et mettre à disposition;

- encourager les agents à mettre en commun les données, les savoirs faire sur un outil collaboratif (GDo) afin de leur faire prendre conscience des perspectives qu'offre la démarche qualité en terme de capitalisation et de diffusion des connaissances ;

- au niveau collectif, favoriser l'apprentissage sur le terrain permettra de capturer les savoirfaire, l'expérience des autres et de s'approprier professionnellement ces connaissances avec le temps;

- communiquer, présenter simplement les travaux qui sont réalisés au sein de l'Unité. Certains ont évoqué des modèles, ce qui peut être une des solutions à mettre en place avec des outils collaboratifs simples (wiki, blog).

Il existe au sein des projets de recherche des connaissances créées pendant le processus de recherche qui reste peu capitalisées et pour lesquelles il n'existe pas d'outil. Il serait intéressant pour l'Unité de se pencher sur ces connaissances afin de voir ce qu'elle pourrait définir en terme de moyen pour les appréhender, puis, ce qu'elle peut partager et avec qui.

\section{CONCLUSION}

Dans le cadre de l'étude effectuée, nous avons établi un certain nombre de résultats qui nous ont permis de confirmer qu'au sein de l'URFM les connaissances se partagent. Les résultats obtenus à l'issue des deux enquêtes que nous avons conduites ont permis de confirmer nos hypothèses de départ. Ils montrent que c'est principalement au moyen des outils mis à leur disposition, et qu'ils ont l'habitude d'utiliser au sein des réseaux, projets et communautés que les agents partagent les connaissances, mais aussi par le biais de rencontres formelles et informelles. Les connaissances explicites sont faciles à partager 
car elles sont réinscriptibles et transmissibles au moyen de supports.

En revanche, le partage reste difficile pour une partie des connaissances tacites qui ne peuvent pas se convertir en connaissances explicites, notamment certaines connaissances scientifiques et celles dites "artisanales", car elles ne peuvent être retranscrites. Elles ne peuvent, en effet, se partager et se transmettre efficacement que par voie orale au cours de rencontres ou sur le terrain, en face à face, par observation, par formation ou par apprentissage. Quelques pistes ont cependant été identifiées pour améliorer le partage des connaissances.

La capitalisation des connaissances est un point important. Elle permet de les identifier, les collecter, les codifier, les centraliser et les diffuser. La démarche qualité pourrait, pour sa part, contribuer à la capitalisation des connaissances techniques et administratives ; cependant, elle nécessite des changements de comportement et d'organisation qui, selon notre étude, ne semblent pas vouloir être facilement adoptés par tous. Elle pourrait également être utilisée comme dispositif pour capitaliser les «résultats intermédiaires" créés tout au long des projets de recherche mais pour lesquels il n'existe pas d'outil. Il serait intéressant pour l'URFM de réfléchir collectivement à la mise en œuvre de méthodes ad hoc.

Le fonctionnement de la recherche implique, le plus souvent, une segmentation par projets, et la collaboration avec d'autres partenaires, ce qui induit de nombreuses et indispensables interactions. Celles-ci amènent, au travers de ces réseaux et communautés, à l'échange d'une grande quantité de connaissances et d'informations utiles mais qui ne sont pas transmises à l'ensemble de l'Unité. Il existe des outils de gestion de projets qui offrent les fonctionnalités adaptées au contexte et devraient permettre une diffusion plus large.
Les outils ne font pas tout, cependant: c'est bien à partir de l'individu que toute collaboration prend forme; c'est donc à ce niveau qu'il est nécessaire d'établir des rapports de confiance qui permettront de favoriser la coopération. La communication est donc essentielle, car elle facilite et permet la compréhension des activités réciproques et le partage des connaissances. Au terme de nos analyses, nous avons émis quelques propositions et suggestions qui devraient permettre d'améliorer ces points au sein de l'URFM.

\section{REMERCIEMENTS}

Je remercie François Lefèvre, Directeur de Recherche à l'URFM, de m'avoir permis de réaliser ce travail, ainsi que de son soutien et de sa relecture, de même, Éric Rigolot, actuel Directeur de l'URFM, pour son soutien et sa relecture. Je remercie tous les agents de l'URFM pour leur participation et leur contribution. Je remercie également Patrick Lambert, Ingénieur d'Études, Unité GAFL (Unité Génétique et Amélioration des Fruits et Légumes) pour sa relecture.

\section{Références bibliographies}

ABBA, H., LELEU MERVIEL, S. (2010), "Communication et confiance au secours de la refonte du système d'information d'une entreprise de services», Les cahiers du numérique, 2010/4 Vol. 6, p. 77-110.

ALAVI, M., LEIDNER, D.E. (2001), «Knowledge Management and Knowledge Management Sytems: Conceptual Foundations and Research Issues », MIS Quaterly, March, Vol. 25, No 1, 107-137,

ALTER, N. (2009), Donner et prendre, La coopération en Entreprise, Édition La découverte, pp. 11-53

BEGAULT, B. (2007), «Usages et pratiques de la publication électronique des résultats de la recherche. Le cas des sciences de 
I'ingénieur », Document numérique (Vol. 10), p. 47-61.

BELIN, O. (2007), «Une définition mixte de I'organisation: une intentionnalité théorique et méthodologique», Communication et organisation, No31, pp. 180-195.

BERNOUX, P. (2009), La sociologie des organisations, Édition Points-Essai, Nouvelle édition, Paris, pp. 235, 269.

BLANCHET, A., GOTMAN, A. (2010), L'enquête et ses méthodes: I'entretien, Édition Armand Colin, $2^{\circ}$ édition, Paris.

BOURDIEU, P. et WAQUANT, L.D.J. (1992), Réponses, pour une anthropologie réflexive, Éditions du Seuil, Paris.

BOURDIEU, P. (1976), «Le champ scientifique», in: Actes de la recherche en sciences sociales, Vol. 2, n² 2-3, juin 1976, La production de l'idéologie dominante, pp. 88-104.

CHARTRON, G. (1997) Nouveaux modèles pour la communication scientifique?, Journées SFSICENSSIB, Villeurbanne, 19-20 novembre 1997, «Une nouvelle donne pour les revues scientifiques?», en ligne http://www.enssib.fr/eco-doc/ [Consulté le 29/08/2013].

CROZIER, M., FRIEDBERG, E. (1977), L'acteur et le système, Éditions du Seuil, Paris.

DIAZ, F. "L'observation participante comme outil de compréhension du champ de la sécurité", Champ pénal/Penal field [En ligne], Vol. || | 2005, URL: http://champpenal.revues.org/79; DOI: 10.4000/champpenal.79, consulté le 1 er septembre 2015 .

FABRE, I. et GARDIES, C. (2008), «L'accès à I'information scientifique numérique: organisation des savoirs et enjeu de pouvoir dans une communauté scientifique», Sciences de la société, $\mathrm{n}^{\circ} 75$, pp. 85-99.

FERRARY M. et PESQUEUX Y. (2006), Management de la connaissance: knowledge management, apprentissage organisationnel ef société de la connaissance, Economica, Paris, p. $16,18$.
FOX, S. (2000). "Communities of practice, Foucault and Actor-Network theory», Journal of management Studies, 37(6), pp. 853-867.

FRIEDBERG, E. (1997), Le pouvoir et la règle. Dynamique de l'action organisée, Éditions du Seuil, $2^{\mathrm{e}}$ édition.

GENELOT, D. et LEFEVRE, V. (2000), Qu'est-ce que le knowledge management? Knowledge Management, Éd. Arts et métier, Dunod.

GUICHARD, E. (2002) L'internet: mesures des appropriations d'une technique intellectuelle, Thèse de doctorat de l'EHESS, Paris.

DODET, M., PIEDAlLU, M.A. (2009), «La démarche qualité à I'Inra», in GRANIER, $C$., MAS, L.Y., FINOT, L., ARNOUX, B., PASQUALINI, N., DOLLE, V., La démarche qualité dans la recherche publique et l'enseignement supérieur, Versailles Cedex, Editions Quæ, Update Sciences \& Technologies, 2009, pp. 33 à $42-121$ à 139.

GRUNDSTEIN M. et RESENTHAL-SABROUX C. (2001), Management des connaissances: vers un «contrat» de l'échange de l'information et du partage des connaissances dans l'entreprise?, Paris: Press-Club de France.

KAROUI M. ef DUDEZERT A, (2010) «La collaboration centrée sur le partage de connaissances et de l'information au sein des équipes virtuelles", Revue de littérature et perspectives de recherche, Actes de la $15^{\text {e }}$ conférence de l'Association Information et Management (AIM), La Rochelle, Mai 2010.

LATOUR, B. (1995), Le métier de chercheur regard d'un anthropologue, INRA.

LATOUR, B. et WOOLGAR, S. (1988), La vie de Laboratoire. La production des faits scientifique, Édition La découverte, Paris.

MEYRIAT, J. (1983), «De la science de I'information aux métiers de l'information», Schéma et schématisation, $n^{\circ} 19$, p. 65-74.

MORIN, E. (1994), "Sur l'interdisciplinarité », Bulletin Interactif du Centre International de Recherches et Études transdisciplinaires, $n^{\circ} 2$, 1994. 
NONAKA I. et TAKEUCHI H. (1995), The Knowledge-Creating Company - Oxford University Press Inc, New York.

NONAKA I., TAKEUCHI H., INGHAM, M. (1997), La connaissance créatrice, la dynamique de I'entreprise apprenante, De Boeck Université, Paris.

PERRIN, A. (2008), "La valorisation des pratiques de management des connaissances, outils de mesure et mesure des outils», in L. Escaffre, P. Ngobo (Eds.), Capital immatériel et performance de I'entreprise, Presses de I'Université, Angers.

PICHOT, C. et al., Solutions pour le partage de données et d'outils dans le Système d'Information $d^{\prime}$ AnaEE France, Atelier IC et Agriculture, 25 Journées Francophones de I'Ingénierie des Connaissances, Clermont Ferrand, 13 mai 2014, http://www.irit.fr/CMS-DRUPAL7/IC2014/sites/ irit.fr.CMS-DRUPAL7.IC2014/files/5-PICHOT.pdf [Consulté le 29/07/2014].

PIGNARD-CHEYNEL N. (2005), «L'édition de revues scientifiques: une forme de marchandisation de la diffusion des connaissances», Sciences de la Société, $n^{\circ}$ 66, 2005.

POLANYI, M. (1967), The Tacit Dimension, Doubleday Anchor Book, New York.
PRAX, J.Y. (2000), Guide du knowledge management, Édition Dunod, Paris.

REIX, R. (1995), «Savoir tacite et savoir normalisé dans l'entreprise», Revue Française de Gestion, 1995, 105, pp. 17-28. p.

RENZL, R. (2008), Trust management and knowledge sharing: The mediating effects of fear and knowledge documentation, Omega, 36, pp. 206-220

SELLIN C. K., Des organisations centrées processus aux organisations centrées connaissance: la cartographie de connaissances comme levier de transformation des organisations. Le cas de la démarche de "Transfert de Savoir-Faire» chez Total, Thèse de doctorat en sciences de gestion, École Centrale Paris, 2011.

VINCK D., JAIME A., GARDONI M., MOSCA J., "La démarche qualité cadre de la capitalisation des connaissances dans les organismes de recherche", Actes de colloque, Autrans, 22-23 janvier 2004.

WENGER, E. (1998), Communities of Practice: learning, meaning and identity, Cambridge University Press. 